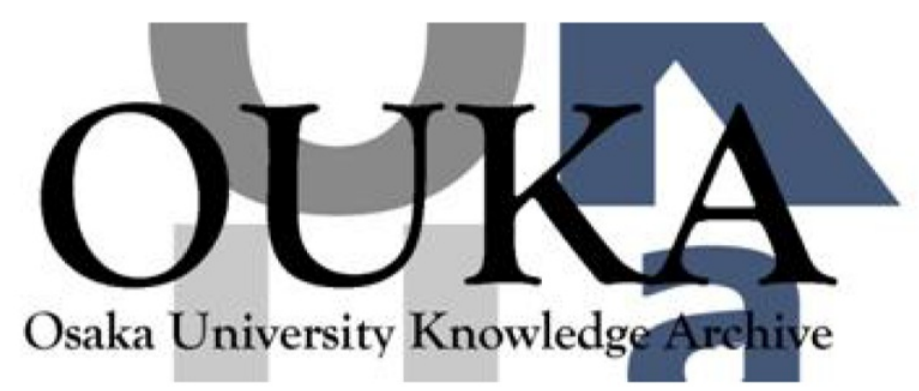

\begin{tabular}{|c|l|}
\hline Title & Basic and integrated studies for fast ignition \\
\hline Author(s) & Tanaka, K.A.; Kodama, R.; Mima, K. et al. \\
\hline Citation & Physics of Plasmas. 10(5) p. 1925-p. 1930 \\
\hline Issue Date & $2003-05$ \\
\hline oaire:version & VoR \\
\hline URL & https://hdl. handle. net/11094/2971 \\
\hline rights & \\
\hline Note & \\
\hline
\end{tabular}

Osaka University Knowledge Archive : OUKA

https://ir. Library. osaka-u. ac. jp/

Osaka University 


\section{Basic and integrated studies for fast ignition ${ }^{a)}$}

K. A. Tanaka, ${ }^{\text {b) }}$ R. Kodama, K. Mima, Y. Kitagawa, H. Fujita, N. Miyanaga, K. Nagai,

T. Norimatsu, T. Sato, Y. Sentoku, ${ }^{\text {c) }}$ K. Shigemori, A. Sunahara, T. Shozaki, M. Tanpo,

S. Tohyama, T. Yabuuchi, J. Zheng, and T. Yamanaka

Institute of Laser Engineering, Graduate School of Engineering, Osaka University, Suita, Osaka, Japan

P. A. Norreys and R. Evanse

Rutherford Appleton Laboratory, Oxfordshire OX11 OQX, United Kingdom

M. Zepf

Queens University, Belfast BT7 1NN, United Kingdom

K. Krushelnic

Imperial College, London SW7 2BZ, United Kingdom

A. Dangor

Imperial College, London SW7 2BZ, United Kingdom

R. Stephens

General Atomic, San Diego, California 92186

S. Hatchett, M. Tabak, and R. Turner

Lawrence Livermore National Laboratory, Livermore, California 94550

(Received 14 November 2002; accepted 6 February 2003)

Basic and integrated studies are conducted on fast ignition (FI) using various large laser systems. A Peta watt (PW) laser system is used to study the basic elements relevant to FI and can also be injected to a compressed core to test the FI integrated experiment when coupled with a GEKKO twelve laser beam system. Using a spherical target inserted with a Au cone guide for the PW laser pulse, an imploded core is heated up to $1 \mathrm{keV}$ resulting in neutron increase 1000 times more than that without heating pulse. Details of the implosion are examined at the Omega laser system of this type target with indirect implosion scheme and are compared with simulation results. LASNEX simulation indicates that a $400 \mathrm{~g} / \mathrm{c} . c$. high density core could be achieved with this scheme at $1.8 \mathrm{MJ}$ laser input. (C) 2003 American Institute of Physics. [DOI: 10.1063/1.1567722]

\section{INTRODUCTION}

After a chirped pulse amplification technique was invented in the mid-1980s, tabletop short pulse laser systems became popular in many scientific fields. Once ultrashort pulse systems with a large energy became available, a fast ignition (FI) scheme was proposed for examination. ${ }^{1}$ FI scheme separates the laser into two systems: one for implosion and the other for heating the imploded fuel core. Since the laser energy required just for implosion is much smaller than that for the central ignition scheme, a high gain could be expected for the FI if the requirement stays reasonable for heating energy. An ultra-intense laser with several tens of kilojoules energy is assumed to transfer energy required for ignition before the core disassembling from the outside when a high-density fuel core is formed with a laser for spherical compression. A total laser energy of a few hundred kilojoules may achieve a gain 100. Transferring the FI energy with an ultra-intense laser pulse through a large plasma corona surrounding the high-density core, a hole boring prior to the

\footnotetext{
a) Paper CI1 3, Bull. Am. Phys. Soc. 47, 55 (2002).

${ }^{b}$ Invited speaker. Also at the Graduate School of Engineering, Osaka University.

${ }^{c}$ Present address: General Atomic, San Diego, CA 92186.
}

ultra-intense laser pulse may be necessary as an optical guide to the core. A relativistic laser self-focusing may also transfer the energy through the corona. However nonlinear laserplasma interactions may have to be studied thoroughly in order to reveal the validity of the above-mentioned ultraintense laser pulse injection. The interaction studies include stimulated Raman scattering, ${ }^{2}$ laser light filamentation and/or self-focusing, ${ }^{3}$ an ultra-intense laser transfer efficiency through the corona, ${ }^{4}$ hot electron energy transport, etc. The idea of using a Au cone inserted into a spherical shell avoids all the interaction processes caused by an ultra-intense laser with a corona plasma, since the Au cone works as a guide of the ultra-intense laser pulse.

We have conducted the basic and integrated studies for FI. The basic study includes details of hot electron production with an ultra-intense laser pulse. The integrated includes a model FI experiment, which shows an enforced heating of a core with a PW (Peta watt) ultra-intense laser pulse. Synchronized to the maximum compression, an ultra-intense laser pulse is focused into the tip of the cone at a high intensity of $10^{18}-10^{20} \mathrm{~W} / \mathrm{cm}^{2}$ to produce hot electrons for core heating. If a core is formed just in front of the cone tip, then hot electrons can heat it without too much energy loss. Also included is a study of Au cone shell implosion that is of 
importance to judge if a high enough density could be achieved at an ignition experiment even with nonspherical implosion with a Au cone.

\section{LASER SYSTEMS}

A PW laser system has been constructed in the same laser bay of the GEKKO 12 laser beam system at the Institute of Laser Engineering, Osaka University. The PW system has a $50 \mathrm{~cm}$ beam diameter and can deliver up to $500 \mathrm{~J}$ on-target within $0.5 \mathrm{ps}$. at $1053 \mathrm{~nm}$ with a $5 \mathrm{~nm}$ bandwidth. A Nd:glass oscillator is used at a $5 \%$ stability. The front-end section has an optically parametric chirped pulse amplifier $(\mathrm{OPCPA})^{5}$ whose output is $20 \mathrm{~mJ}$ with a $9 \mathrm{~nm}$ bandwidth. After four final Cassegrain-type final amplifiers with a 8 $\mathrm{Nd}$ :glass disks, a pulse width is $1.5 \mathrm{~ns}$. with a $3 \mathrm{~nm}$ bandwidth and has a ring shape of $315 \mathrm{~cm}$ outer and $135 \mathrm{~cm}$ inner diameter. The filling factor of the beam is $62 \%$. The amplified PW beam is guided into a large compressor chamber which has four $1 \mathrm{~m}$ grating with 1480 lines $/ \mathrm{mm}$. The transmission efficiency is $60 \%$ through the compressor. The PW pulse is focused with an $f / 7.6$ off-axis parabola mirror of 3.8 $\mathrm{m}$ focal length to a $30 \mu \mathrm{m}$ focal spot. Because of the OPCPA system, a pre-pulse intensity is at an order of $10^{-8}$ compared to the main.

The GEKKO laser system has 12 green beams whose oscillator takes a part of the PW front-end light. The synchronization is within $10 \mathrm{ps}$ between the PW and GEKKO pulses because both systems use the same origin of light. The GEKKO laser system delivers $2.5 \mathrm{~kJ}$ at $532 \mathrm{~nm}$ onto a target with random phase plates with a $1.2 \mathrm{~ns}$ quasi-square pulse shape. The two laser systems were used to conduct a FI model experiment where the PW laser pulse is injected through a Au cone to heat a compressed core imploded by the GEKKO laser beams.

The OMEGA laser system has 60 beams at $351 \mathrm{~nm}$ at the Laboratory for Laser Energetics, University of Rochester. ${ }^{6}$ This laser system has been used as a national user's facility program to examine the hohlraum implosion of a polymer shell inserted with a Au cone. Forty UV beams irradiate the hohlraum to implode a $500 \mathrm{~mm}$ shell at $14 \mathrm{~kJ}$. Twenty UV beams are used to x-ray backlight the imploded core at $7 \mathrm{~kJ}$.

\section{Au CONE PERFORMANCE}

Ultra-intense laser focusing is studied of a cone using three-dimensional particle in cell (PIC) simulation. ${ }^{7}$ The simulation conditions are such that a laser intensity is 4 $\times 10^{18} \mathrm{~W} / \mathrm{cm}^{2}$, the pulse width is $20 \mathrm{fs}$. The dimension of the cone is $6.5 \mu \mathrm{m}$ deep with a $3 \mu \mathrm{m}$ tip with a $54 \mathrm{deg}$ opening angle. The material is deuteron of five times the laser critical density $\left(5 n_{c}\right)$. The simulation box is $14 \mu \mathrm{m}$ $\times 9 \mu \mathrm{m} \times 9 \mu \mathrm{m}$. When the ultra-intense laser pulse comes into the cone, the electric field is focused and is concentrated toward the cone tip. The intensity amplification is clearly observed in Fig. 1, where the $E$ field at the perpendicular to the polarization plane is shown at $t=50$ fs. Figure 1 shows the intensity plot indicating that the amplified intensity reaches 20 times the original intensity. In the simulation hot

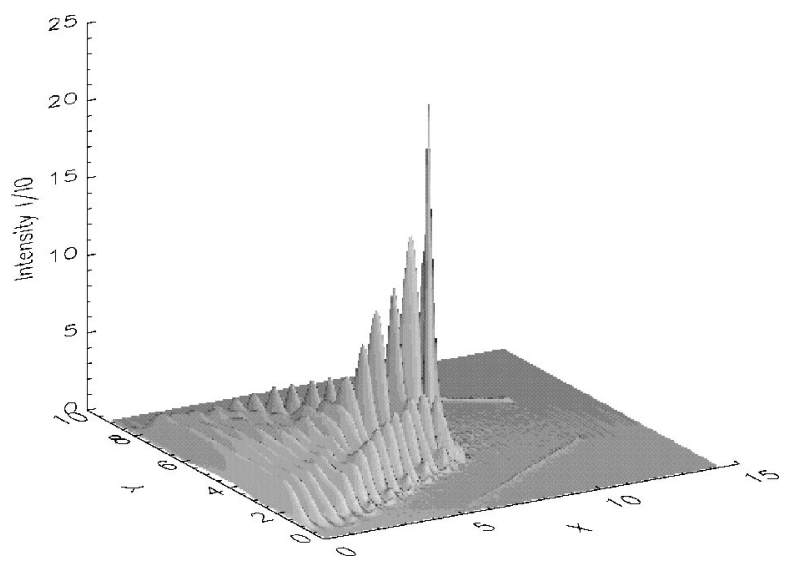

FIG. 1. The $E$ field is shown of an ultra-intense laser pulse incident into a $54^{\circ}$ cone perpendicular to the polarization in the three-dimensional particle in cell (PIC) simulation. The concentration of the $E$ field along the cone axis is clearly shown. 20 times increase is indicated over the original intensity $4 \times 10^{18} \mathrm{~W} / \mathrm{cm}^{2}$. The scale of $X$ and $Y$ directions are normalized by the laser wavelength $1 \mu \mathrm{m}$. The figure is at $t=100 \mathrm{fs}$, while the ultra-intense laser pulse is $20 \mathrm{fs}$. The simulation is calculated in $14 \times 9 \times 9 \mu \mathrm{m}$ box.

electron current also runs along the surface wall and tends to increase its intensity toward the tip. From the simulation it is expected that the ultra-intense laser pulse may be focused effectively without any special optics. A low intensity halo in the focal spot may be shifted toward the center propagating along the cone axis. The pointing accuracy of the laser may be relaxed if the ultra-intense laser pulse is injected into a $\mathrm{Au}$ cone even with a slight displacement and is focused along the cone wall.

A distinctive difference is observed in the hot electron spectrum when the PW laser pulse at $190 \mathrm{~J}$ irradiates two different targets. The vacuum focus intensity is $5 \times 10^{18}$ $\mathrm{W} / \mathrm{cm}^{2}$. Figure 2(a) shows the hot electron spectra for the two cases. The left spectrum is for an aluminum plane target, while the right is for a $\mathrm{Au}$ cone. The $\mathrm{Au}$ cone has a $30^{\circ}$ opening with a 5- $\mu \mathrm{m}$-thick tip of $50 \mu \mathrm{m}$ diameter. The $\mathrm{Al}$ target was used to check the focusability of the PW laser beam before the Au cone experiments whose $\mathrm{x}$-ray thermal emission was matched well to an x-ray charge-coupled

(a)

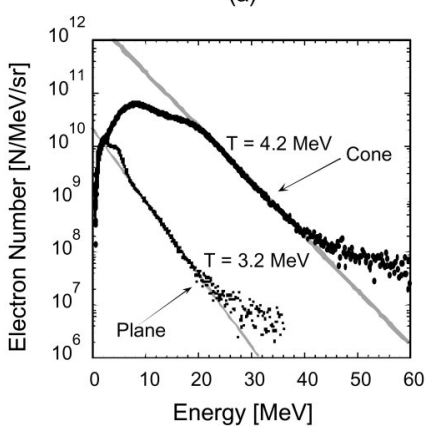

FIG. 2. (a) Hot electron spectra: one left for an Al planer, the other right for a Au cone $\left(30^{\circ}\right)$. The slope temperature for the Au cone becomes higher than the one for the Al plane. Also the number of electrons is increased. These spectra are measured at $20^{\circ}$ (forward direction) from the PW laser axis. (b) Comparison between the $\mathrm{Au}$ cone and $\mathrm{Al}$ plane is shown in the angular distribution. The spectra are measured at both $20^{\circ}$ and $40^{\circ}$. The electron flux is collimated and increases with the Au cone. 
device/pin-hole camera system. According to our many shot experience the number of the electrons observed by electron spectrometers did not depend on the target materials. The horizontal and vertical axes are the energy $(\mathrm{MeV})$ and the number $(/ \mathrm{MeV} / \mathrm{sr})$ of hot electrons. Hot electrons are measured with electron spectrometers using imaging plate as an electron detector with $1.5 \mathrm{kG}$ magnet pair at a $40^{\circ}$ observation angle from the PW laser axis and $4.5 \mathrm{kG}$ magnet pair at $20^{\circ}{ }^{8}$ Both spectrometers have a solid angle of $10^{-5} \mathrm{sr} \mathrm{Al}$ plane target case shows a $3.2 \mathrm{MeV}$ slope temperature and the peak electron number is about $10^{10} / \mathrm{MeV} / \mathrm{sr}$. When the $\mathrm{PW}$ pulse is injected into the Au cone, the slope temperature is more than $4 \mathrm{MeV}$ and the number increases close to $10^{11} / \mathrm{MeV} / \mathrm{sr}$. The spectral width appears broadened compared to that of the Al plane. The increase of the electron number is also shown in Fig. 2(b) in the angular plot. In the simulation the hot electrons are created along the Au cone surface and are guided toward the tip. The guiding was conducted with the existence of a large $B$ field (50 MG) across the Au wall generated with the hot electron current. Actual laser intensity increase may depend on the focal spot structure in the experiment and is difficult to compare directly with the PIC simulation. The ultra-intense laser beam may be reflected on the Au surface in the experiment since the beam irradiates the Au wall at an angle much shallower than the simulation, which may lead to a higher laser intensity at the tip. These hot electrons tend to be transferred in a solid target with a single or few filaments when ultra-intense laser pulse carries more than $100 \mathrm{TW}$ power, resulting in a good heating device of a core. ${ }^{9}$ The angular distribution of hot electrons measured by limited number of electron spectrometers is rather reproducible from shot to shot. The distribution has also been proven to be reproducible with the use of imaging plate directly placed behind planar targets in a separate experiment. ${ }^{10}$ Thus the use of a Au cone not only works to block the corona plasma but also works to improve the ultraintense laser focused beam quality. At the tip the electron flux is amplified. The increase of hot electron number and temperature is fully consistent with the PIC simulation. The level of tolerance for mispointing and displacement of the high-power beam is now under investigation. ${ }^{11}$

\section{INTEGRATED FI MODEL EXPERIMENT}

A Au cone inserted on a plastic shell is imploded and is heated to model enforced heating of an imploded high density core using both GEKKO and PW laser systems. ${ }^{12}$ The gold cone has a $30^{\circ}$ opening angle with a $5 \mu \mathrm{m}$ tip thickness at $50 \mu \mathrm{m}$ diameter. The shell is $500 \mu \mathrm{m}$ in diameter with $7-\mu \mathrm{m}$-thick wall and its material is $\mathrm{CD}$ (deuterated plastic). A typical target picture is shown in Fig. 3. Nine laser beams of the GEKKO irradiate a CD shell for implosion with $1.2 \mathrm{~ns}$ pulse at a $532 \mathrm{~nm}$ wavelength width of a flat top shape at an energy of $2.5 \mathrm{~kJ}$. Random phase plates are inserted in all the beams. Implosions are also simulated with a onedimensional fluid code. The implosion timing is monitored with an X-ray streak camera and agrees well with the simulation. At around the maximum compression, the PW laser of $300 \mathrm{~J}$ in $0.6 \mathrm{ps}$ is injected into the Au cone tip. According to

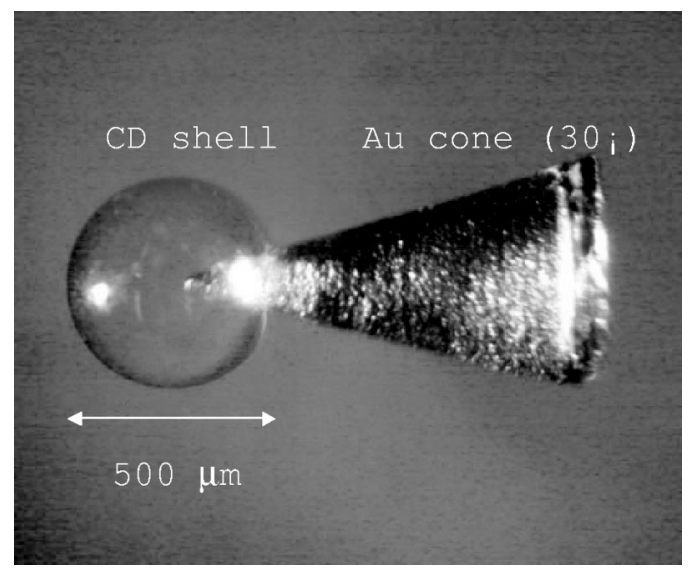

FIG. 3. CD shell inserted with a Au cone. The opening angle of the cone is $30^{\circ}$. The shell diameter is $500 \mu \mathrm{m}$. The tip of the cone is $50 \mu \mathrm{m}$ away from the shell center. The shell is first driven for implosion with 9 laser beams of $1.2 \mathrm{~ns}$ pulse. At the maximum compression the PW ultra-intense laser pulse is focused into the cone at $300 \mathrm{~J}$ in $0.6 \mathrm{ps}$. The generated electrons at the tip are used to fast heat the core.

our simulation, the Au cone tip survives to the maximum compression. The imploded core is created at about $50 \mu \mathrm{m}$ away from the tip, which gives a very good coupling of hot electrons to the core. The compression process is also monitored with an $\mathrm{x}$-ray framing camera making use of the x-ray backlight of Pt at $2 \mathrm{keV}$, which could give an estimation of the core density. The diameter and the density of the core are $30-50 \mu \mathrm{m}$ and $50-100 \mathrm{~g} / \mathrm{cm}^{3}$, respectively. The density is reduced from fitting the back lighting data with a simulation. The PW injection is confirmed as a clear $\mathrm{x}$-ray signal with the $\mathrm{x}$-ray streak camera.

The neutron spectrum was measured at $42^{\circ}$ from the laser axis at both 3 and $4.5 \mathrm{~m}$ distance from the target. The typical neutron spectrum is shown in Fig. 4, where the width of the spectrum is $90 \mathrm{keV}$ at the $2.45 \mathrm{MeV}$ DD fusion energy. The width corresponds to the ion temperature of $800 \mathrm{eV}$ $(+0.1 \mathrm{keV})$. This signal is purely due to thermal neutrons. When the process includes a beam neutron reaction, the spectrum shows a much broadened one due to the Doppler effect of moving ion beams. ${ }^{13}$ Figure 5 shows a graph of

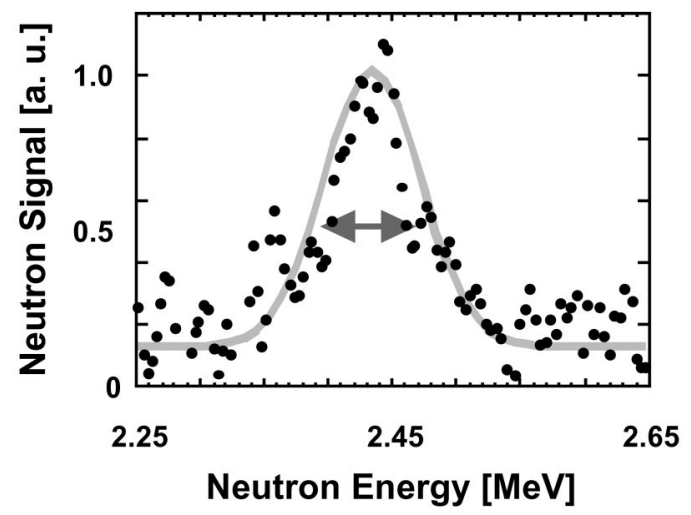

FIG. 4. Neutron time of flight signal. The width measured at $2.45 \mathrm{MeV}$ is about $90 \mathrm{keV}$, which corresponds to an ion temperature $800 \mathrm{eV}$. Two scintillation detectors are set at $42^{\circ}$ at 3 and $4.5 \mathrm{~m}$ distances from the target. Time coincidence is taken to identify the neutron signal. 


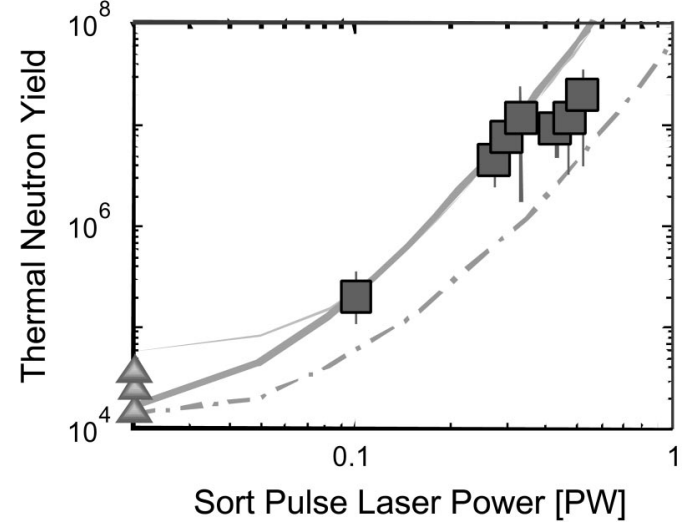

FIG. 5. Neutron number vs fast heating laser power. The triangles show the data without fast heating. The squares show the data with fast heating. The neutron increase is 1000 times with the heating compared to the ones without it. The fitted curves are the ones with $30 \%$ and $15 \%$ coupling efficiencies from the ultra-intense laser to the core heated energy.

neutron yield versus fast heating laser power. $10^{4}$ neutrons are observed just from implosion without the PW heating. When the ultra-intense laser pulses at $0.5 \mathrm{PW}$ are injected into the cones at max compression, the neutron yield increased to almost $10^{7}$. In the previous study of fast heating at a 100 TW laser power, a similar compressed core was created and was estimated to have a $300 \mathrm{eV}$ plasma temperature. In this experiment the temperature rise from 300 to 800 $\mathrm{eV}$ is also consistent with the 1000 times neutron increase. From this graph one can see that the scaling from the no heating to the $0.1 \mathrm{PW}$ is extended almost to $0.5 \mathrm{PW}$. The fitting curve is the simple estimation of neutron production from a core heated with a total coupling efficiency $30 \%$ (curve above) and 15\% (curve below) from the ultra-intense laser. At a PW level, which is within a factor 2 from the present data, neutron increase of $10^{4}$ to $10^{5}$ would be possible.

\section{IMPLOSION OF SHELL WITH Au CONE, SCALED DOWN FROM AN IGNITION DESIGN}

The implosion of a capsule with an inserted Au cone has been studied in a hohlraum geometry using the Omega laser system at the Laboratory for Laser Energetics, University of Rochester. A polymer shell of $500 \mu \mathrm{m}$ diameter with a 50$\mu$-m thick wall has a Au cone of $70^{\circ}$ opening angle as shown in Fig. 6. The tip of the cone is placed at $12 \mu \mathrm{m}$ away from the target center. This shell is driven with a soft $\mathrm{x}$-ray pulse, which is slowly rising and has a peak radiation temperature of $\sim 180 \mathrm{eV}$ at $2.3 \mathrm{~ns}$. Forty laser beams at $351 \mathrm{~nm}$ are injected to obtain the implosion with $14 \mathrm{~kJ}$ energy. The implosion is monitored with an X-ray backlighting technique using a Fe $6.7 \mathrm{keV}$ backlighting source. The backlight source Fe plate ( $7 \mu \mathrm{m}$ thick) is attached on the hohlraum case as a window normal to the cone and hohlraum axes. On the opposite side of the $\mathrm{Fe}$ an observation window $(\mathrm{CH} 50 \mu \mathrm{m}$ coated with $0.15 \mu \mathrm{m} \mathrm{Ta})$ is attached to observe the $\mathrm{x}$-ray backlit image of the core. Twenty laser beams are focused on the Fe plate to transmit the $\mathrm{x}$-ray backlight source toward the

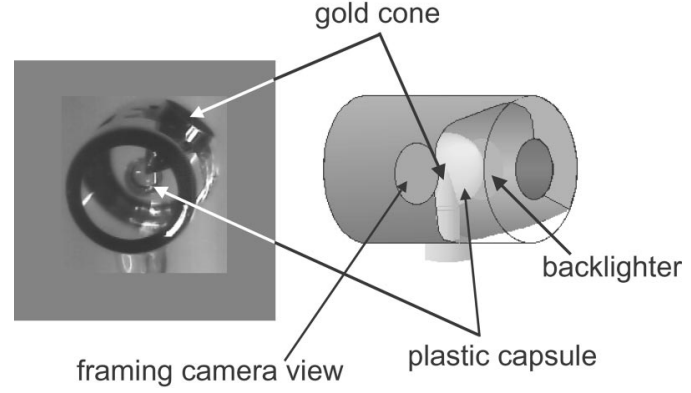

FIG. 6. Hohlraum target used to study the shell implosion with a Au cone. A $510 \mu \mathrm{m}$ polymer shell is held at the center of Au hohlraum. The schematic view of the target is also shown in the right. A Fe window is attached to take an X-ray backlight of imploded images of the core at $6.7 \mathrm{keV}$.

imploding core at $7 \mathrm{~kJ}$. The implosion process is recorded with a 70 ps. framing camera with a $10 \mu \mathrm{m}$ spatial resolution.

These indirectly driven implosions at LLE were simulated in two-dimensional (axial symmetry) with the ICF code LASNEX. ${ }^{14}$ We used Lagrangian hydrodynamics, rezoning as needed to untangle the mesh, and modeled radiation transport with multi-group diffusion. The gold cone boundary on the capsule side was treated with a free-slip boundary condition. The other (inner) boundary of the gold cone was treated as a free surface. The time and frequency dependent $\mathrm{X}$-ray drive incident on the capsule was taken from hohlraum simulations previously used to model conventional indirectdrive implosions with similar hohlraum dimensions, capsule sizes, and laser drive profiles. Figure 7 shows a comparison of the imploded cores from (a) the experiment and (b) the simulation. There is an overall similarity between the two. Both cores have diameters around 60-70 $\mu \mathrm{m}$, tend to show a round shape toward the soft $\mathrm{x}$-ray irradiation side and a somewhat flat structure on the $\mathrm{Au}$ tip side. The obtained densities are $20 \mathrm{~g} / \mathrm{c} . \mathrm{c}$. for the observed and $27 \mathrm{~g} / \mathrm{c} . \mathrm{c}$. for the

\section{X-ray backlight \\ @ $\mathrm{hv}=6.7 \mathrm{keV}$}

(a) Experiment

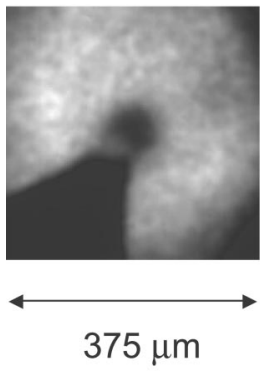

$\rho=20$ g/c.c. (b) Simulation

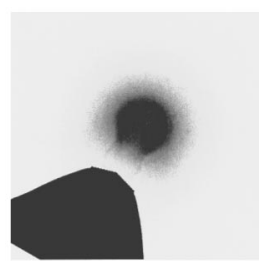

$\rho=27$ g/c.c.
FIG. 7. (a) Observed compressed core backlit with $6.7 \mathrm{keV} x$ ray. The size is about $70 \mu \mathrm{m}$ diameter. (b) Simulated compressed core for $6.7 \mathrm{keV}$ x-ray backlight. The size is about $60 \mu \mathrm{m}$. The overall similarity is seen, but there is a difference at the tip area. Au plasma ablated at early time seems to be extended toward the core. The simulation does not show the Au plasma ablation since $M$ line of $\mathrm{Au}(2.2 \mathrm{keV})$ is not included. 
simulation. The density is estimated from the x-ray opacity evaluation through the core. There are, however, differences between the two images at the space between the Au tip and the core. In Fig. 7(a) the gap between the tip and the core appears to be filled up with something opaque to the $6.7 \mathrm{keV}$ $\mathrm{x}$ ray while the simulated image shows a clear gap. ${ }^{15} \mathrm{We}$ believe the opacity seen in the experiment is due to gold plasma ablated from the tip of the cone by hard $\mathrm{x}$ rays, principally $\mathrm{Au} M$-line radiation, from the laser spots on the hohlraum wall. The model $\mathrm{x}$-ray drive included the nonthermal $M$-line radiation from the gold hohlraum. In the simulations these high energy $x$ rays penetrate the capsule and heat the tip of the cone, causing gold plasma to ablate out into the void inside the capsule shell. Later, as the capsule collapses, plastic plasma blown off the inside of the shell by shock waves impinges on the gold plasma with a pressure gradient that tries to push the gold plasma back toward the cone tip. Since the gold plasma at the interface is denser than the plastic plasma, the interface is Rayleigh/Taylor unstable. This instability, however, is not captured by our simulations - partly because of the free-slip boundary condition we imposed, and mostly because the interface was not "seeded" with any perturbations. Therefore, in the simulations the gold plasma is pushed back with the result that there is no significant opacity in the space between the core and the cone. In reality we expect the interface to be quite perturbed with the result that the gold plasma would not be pushed back toward the cone but would instead be mixed with the plastic plasma in the interface region resulting in the opacity seen in the experiment. This effect (which could be quite detrimental to full scale fast ignition) should not occur if the capsule is directly driven. An experimental test of this hypothesis is currently being undertaken.

We have modeled indirectly driven full ignition-scale targets with the LASNEX code in a manner like that described earlier for the LLE targets - with the addition of a collimated or diverging beam of electrons emerging from a disc source on the inside of the gold cone and representing the hot electrons driven by the UI laser pulse. Since our interest has been to simply scope out the ignition energetics, no attempt has been made to do realistic electron transport. Instead the electrons are treated by LASNEX's ion beam package-they deposit energy along their paths with $d E / d x$ appropriate for real electron energies and the electron mass but they travel along straight paths. All collective electrodynamic effects (return currents, etc.) are also ignored. The polymer shells in the above-described LLE experiments correspond roughly to a $1 / 5$ scale of a previously designed ignition target ${ }^{16}$ and were intended to begin to verify the ignition target hydrodynamics. The ignition target, incorporating a similar gold cone, is a 2-mm-diam $\mathrm{Be}-\mathrm{Cu}$ shell with a thick DT fuel layer. If the drive is symmetric, the imploded core tends to have a lower density central region, rather similar to that seen in Fig. 8(a). However, we find that a $10 \% P_{1}$ asymmetry in the incident $\mathrm{x}$-ray flux, hotter opposite the cone, results in a more isochoric type core as shown Fig. 8(b). This form should more efficiently absorb a collimated electron beam. In our simulations the areal density and density of imploded DT cores are up to $\rho R=2.2 \mathrm{~g} \mathrm{~cm}^{-2}$ and $\rho=400 \mathrm{~g} / \mathrm{cm}^{-3}$. This

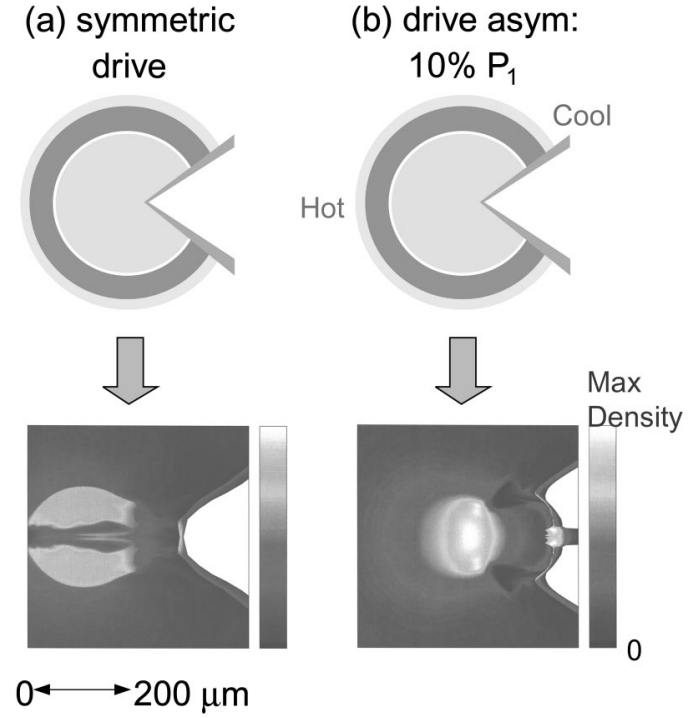

FIG. 8. (a) Simulated compressed core for fast ignition. Drive was symmetric except the solid angle blocked by Au cone on the right. The core shows a hollow structure. $\rho R$ is $2.03 \mathrm{~g} / \mathrm{cm}^{2}$. (b) Simulated compressed core for fast ignition. Drive was asymmetric by $10 \%$ stronger with $P_{1}$ mode on the opposite side of the cone. The core shows a constant density structure. $\rho R$ is $2.10 \mathrm{~g} / \mathrm{cm}^{2}$.

core can be ignited with $30 \mathrm{~kJ}$ of $\left\langle E_{e}\right\rangle \sim 2-3 \mathrm{MeV}$ electrons, if they are collimated within a $40-\mu \mathrm{m}$-diam beam, and yields 27 MJ of output energy. The UI laser energy required to generate these electrons depends on various efficiency factors including the conversion efficiency from laser to hot electrons, the distribution of laser intensities on target, and focusing effects within the cone like those seen in the PIC simulations described earlier. An estimate, assuming 50\% conversion efficiency, is that $\sim 80 \mathrm{~kJ}$ of UI laser might be required.

\section{SUMMARY}

We have shown the basic and integrated studies related to the fast ignition scheme. In the basic the guiding cone is shown to work as a focusing optics of an ultra-intense laser pulse once injected into the cone using a three-dimensional PIC simulation. The intensity increase could be as high as 20 times the original vacuum focus intensity. The increase of hot electron flux is also suggested. In the ultra-intense laser experiment, a large increase of hot electron flux is observed with a $\mathrm{Au}$ cone compared to a case with an $\mathrm{Al}$ plane at 5 $\times 10^{18} \mathrm{~W} / \mathrm{cm}^{2}$. The hot electron temperature increases also from 3.2 to $4.2 \mathrm{MeV}$. Thus the use of Au cone is an appropriate choice for modeling FI experiment in addition to the original idea of avoiding the nonlinear ultra-intense laserplasma interactions in the corona surrounding a high density compressed core.

The integrated experiment is conducted using the GEKKO 12-beam and PW ultra-intense systems, which are perfectly synchronized. A CD shell with a Au cone $\left(30^{\circ}\right.$ opening angle) is imploded, is heated at its maximum compression with the PW ultra-intense laser pulse. Thermal DD neutrons are observed to increase 1000 times with the use of 
the PW enforced heating compared to the case without it. The estimated ion temperature is about $800 \mathrm{eV}$. The heating is in good agreement with a simple estimate of the core heating and is extended from the previous 100 TW FI model experiment to a level close to the PW necessary for an ignition.

The details of the Au cone shell implosion are compared with the LASNEX simulation. The implosion is driven with a soft x-ray pulse with a peak radiation temperature of $180 \mathrm{eV}$. The overall structures of the imploded core show are in agreement with the simulation. There is, however, a difference in the region between the core and the Au tip. The Au plasma appears to be extended to the core much closer than the simulated which is caused with the Au $M$-line ablation in early time. An ignition level target scaled up from the experiment indicates that the areal density and the density of the core could be as high as 2.2 and $400 \mathrm{~g} / \mathrm{cm}^{3}$ and are attained when 1.4 MJ laser is incident into a hohlraum.

\section{ACKNOWLEDGMENTS}

The PW and GEKKO laser operation, target fabrication, diagnostics operation groups are greatly appreciated for the FI model experiment at ILE, Osaka University. We acknowledge the target fabrication at General Atomic. The Omega laser and diagnostics groups are greatly appreciated at LLE, University of Rochester. Parts of this work have been supported by NLUF program at LLE, University of Rochester. Parts of this work have also been supported by the Japan-
United Kingdom, Japan-US collaboration programs of Japan Society for the Promotion of Science as well as the JapanChina including the post doc. Program of Dr. J. Zheng.

${ }^{1}$ T. Yamanaka, Kongo Project Report, Internal Report of ILE, Osaka University, 5 (1977); M. Tabak, J. Hammer, M. Glinsky et al., Phys. Plasmas 1, 1626 (1994).

${ }^{2}$ T. Miyakoshi, M. Jovanovic, Y. Kitagawa et al., Phys. Plasmas 9, 3552 (2002).

${ }^{3}$ K. A. Tanaka, M. Allen, A. Pukhov et al., Phys. Rev. E 62, 2672 (2000).

${ }^{4}$ K. A. Tanaka, R. Kodama, H. Fujita et al., Phys. Plasmas 6, 2847 (1999); R. Kodama, K. A. Tanaka, Y. Kitagawa et al., ibid. 8, 2268 (2001).

${ }^{5}$ D. Strickland and G. Mourou, Opt. Commun. 56, 219 (1985).

${ }^{6}$ J. M. Soures, R. McCrory, C. Verdon et al., Phys. Plasmas 3, 2108 (1996).

${ }^{7}$ Y. Sentoku and K. Mima, submitted to Phys. Rev. Lett.

${ }^{8}$ T. Takahashi, T. Sato, T. Yabuuchi et al., Hoshasen 1, 203 (2002), in Japanese.

${ }^{9}$ Y. Tohyama, R. Kodama, T. Tsutumi et al., Bull. Am. Phys. Soc. 47, 23 (2002); K. Mima et al., Patent No. 2002-211116 (2002); M. Tabak, E. M. Campbell, J. H. Hammer, W. L. Kruer, M. D. Perry, S. C. Wilks, and J. G. Woodworth, Lawrence Livermore National Laboratory Patent Disclosure, IL-8826B, 1997, Lawrence Livermore National Laboratory, Livermore, CA

${ }^{10}$ T. Sato, K. A. Tanaka, R. Kodama et al., ILE Annual Progress Report No. 27, 2001.

${ }^{11}$ R. Kodama and T. Yabuuchi (private communications).

${ }^{12}$ R. Kodama, P. Norreys, K. Mima et al., Nature (London) 412, 7989 (2001); R. Kodama et al., ibid. 418, 933 (2002).

${ }^{13}$ N. Izumi, Y. Sentoku, H. Habara et al., Phys. Rev. E 65, 036413 (2002).

${ }^{14} \mathrm{~J}$. Harte et al., 1996 ICF Annual Report, Lawrence Livermore National Laboratory, Livermore, CA, UCRL-LR-105821-96, 1997, p. 150.

${ }^{15}$ R. Stephens, S. Hatchett, R. Turner et al., Bull. Am. Phys. Soc. 47, 142 (2002).

${ }^{16}$ S. Hatchett, R. Stephens, M. Tabak, and R. Turner, Proceeding of the Fifth Workshop on Fast Ignition of Fusion Targets, Medeira, 18-22 June 2001. 